\title{
THE EFFECT OF DISCRIMINATION TRAINING ON PATTERN EQUIVALENCE IN MONKEYS WITH INFEROTEMPORAL AND LATERAL STRIATE LESIONS*†
}

\author{
Charles M. Butter
}

The University of Michigan

(Received 7 June 1967)

\begin{abstract}
Monkeys with inferotemporal (IT) lesions, monkeys with latcral striate (LS) lcsions and unoperated controls discriminated between two patterns and were tested for pattern equivalence in three experiments. The IT monkeys appeared to utilize salient cues more than control animals did and failed to identify specific pattern features (exps. 1 and 2). When the salient cues were removed (exp. 3) the IT animals gave evidence of normally identifying configurational cues. While two LS monkeys showed deficits like those of the IT monkeys (exps. 1 and 2), they apparently did not identify configurational features (exp. 3). The findings suggest that IT lesions do not impair the capacity to identify visual features, but disrupt stimulus sampling or attentional processes.
\end{abstract}

BILATERAL removal of inferotemporal (IT) cortex in monkeys produces a severe discrimination learning impairment that appears to be restricted to the visual modality [1-3]. While this visual impairment is apparently not due to sensory defects [4-6], the nature of the underlying disturbance is not clear, although the impairment has been investigated in a number of studies (see Mishkin [7] for a review of these studies). The majority of these experiments have employed discrimination learning situations, in which IT monkeys show a retarded rate of learning. In a further analysis of this problem, BuTTER et al. [8] employed stimulus generalization tests to determine whether the effective stimulus for IT monkeys is as specific as it is for their controls, once they have learned to respond to a compound visual stimulus. The IT animals showed abnormally broad generalization gradients, a finding which suggested that these animals were deficient in detecting the less salient features (the specific hue and specific orientation) of the training stimulus. However, once they received the appropriate discrimination training, the IT monkeys showed generalization gradients like those of the control animals, indicating that these animals could utilize the specific features of visual stimuli as well as control animals did. This interpretation of the generalization findings would seem to suggest that the perceptual capacities of IT monkey are intact, but are not evident until the animal is required to attend to particular stimulus features. Further findings from a study employing pattern equivalence testing [9] in part support these conclusions. Thus, once monkeys with IT lesions have learned to discriminate between two forms differing with respect to several cues, they behaved in equivalence testing as though they were deficient in utilizing the cue that was less salient for normal monkeys (configuration) and utilized the more salient cue (position of the form) to a greater

* This research was supported by Grant GB-3127 from the National Science Foundation and Grant MH-06964 from the National Institute of Mental Health.

$\dagger$ The author thanks STEven Doenrman for his assistance in testing the animals. 
extent than unoperated controls did. If, as the findings of BUTTER et al. [8] suggest, IT monkeys can utilize the less salient features of visual stimuli with appropriate training, it might be possible to train IT monkeys to utilize the configurational features of patterns and show normal pattern equivalence with appropriate discrimination training. Such a linding, together with the generalization findings referred to previously, would support the vicw that the IT monkey's deficit in visual learning is due not to an impaired capacity to code visual features, but rather to a failure to utilize potentially effective stimuli in a normal manner.

\section{EXPERIMENT I}

The first experiment was undertaken in order to verify the hypothesis derived from prior results that monkeys with IT lesions are selectively impaired in utilizing the features of visual forms that are less salient for normal monkeys [9]. On the basis of previous equivalence lindings $[9,10]$, it was anticipated that the salient features of the triangle and the circle shown at the top of Fig. 1 would be the cues near the base of the plaques on which the forms are presented (straight vs. curved border), rather than the configuration of the

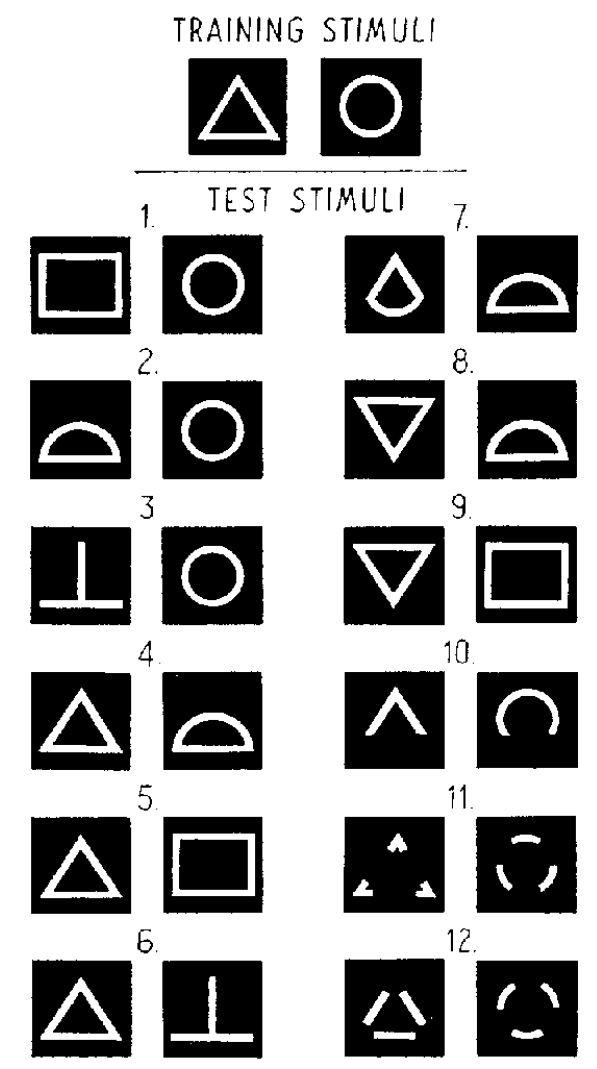

Fig. 1. Stimulus pairs used in discrimination training and in equivalence testing (exp. 1).

forms. Thus, it was expected that IT monkeys would be impaired in utilizing the less salient configurational cue and utilize the more salient "base cue" more than control animals would. These predictions were tested by determining the preferences of IT and control monkeys for the equivalence stimuli shown in Fig. 1 following triangle-circle discrimination learning. 
If the base cue were the most salient feature of the patterns, one might expect control animals to show strong preferences for the left-hand members of pairs 1-3, but only weak preferences for the triangles in pairs $4-6$, in which this cue is absent. In pairs 7-9, the positive base cue (straight line near the base) is in conflict with the positive configurational cue; thus, it was predicted that control animals would prefer the right-hand members of these pairs. Moreover, it was anticipated that the controls would show only slight prelerences for the triangles in pairs 10 and 11 (in which the base cue is absent or partially removed), but stronger preferences for the triangle in pair 12, in which the base cue is present. Furthermore, if the IT animals, compared to their controls, were to utilize the configurational cue less and the base cue more, one might expect them to show abnormally strong preferences for the left-hand members in pairs $1-3$ and 12 and for the right-hand members in pairs 7-9; one might also expect them to show abnormally weak preferences for the triangles in pairs $4-6,10$ and 11 .

\section{METHODS}

\section{Subjects}

The subjects were 12 experimentally naive rhesus monkeys (Macaca mulatta) of both sexes, ranging in weight from $3.7 \mathrm{~kg}$ to $5.1 \mathrm{~kg}$. Four monkeys had bilateral lesions in IT cortex, four operated controls had bilateral removals of lateral striate (LS) cortex, and the remaining four were unoperated controls. Subjects were maintained on a diet of Purina Monkey Chow (45 cal $/ \mathrm{kg}$ per day).

\section{Surgery}

Animals were anesthetised with sodium pentobarbital $(40 \mathrm{mg} / \mathrm{kg})$, and surgery was conducted under aseptic conditions. The surgical procedures were similar to those employed by WILSON and MisHKIN [6]. In brief, one stage bilateral lesions were made by aspiration with a small gauge sucker through openings rongeured in the temporal or occipital bone. The IT lesions were intended to involve all cortex labelled TE in Fig. 2, including the ventral bank of the superior temporal sulcus and extending to the occipitotemporal sulcus on the ventral surface of the temporal lobes. The LS lesions were intended to involve all striate cortex on the lateral surface of the occipital lobes labelled $\mathrm{OC}$ in Fig, 1, including the occipital pole. Since these animals are still being tested in other experiments, the locus and extent of the lesions have not yet been verified. Histological analyses of these brains will be presented in a future report.

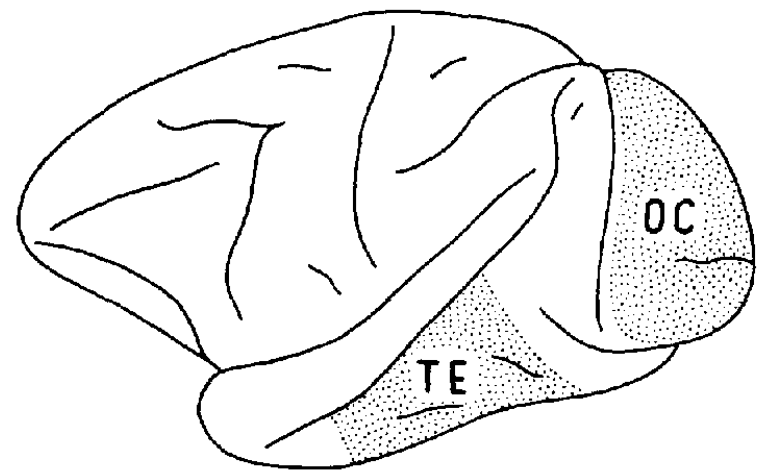

FIO. 2. Schematic view of rhesus monkey brain, showing intended loci of IT lesions (labelled

TE) and of LS lesions (labelled OC). Letters follow VON BONIN and BAILEY's nomenclature [11].

\section{Apparatus}

Animals received discrimination training and equivalence testing in a Wisconsin General Test Apparatus (WGTA). The subject's cage was separated by an opaque screen from an illuminated stimulus compartment. This compartment contained a wooden form board with two food wells, 13 in. apart. When the opaque screen was raised, the monkey could reach through the bars of its cage into the stimulus compartment and displace plaques covering the foodwells in order to obtain food reward. The experimenter could observe the subject's response through a one-way mirror in a second screen. Training and testing were conducted in a darkened room, and outside noises were partially masked by fans. 


\section{Procedure}

In initial training, the subjects learned to displace neutral, gray plaques covering the foodwells in order to obtain half a peanut. They were then trained to discriminate between the triangle and the circle shown at the top of Fig. 1. The patterns were constructed of while paper pasted on gray plaques, 3 in. square. On each trial the two patterns were presented simultaneously over the two foodwells, and responses to the triangle were always rewarded, while responses to the circle were not rewarded. Intertrial intervals were approximately $5 \mathrm{sec}$ in duration, and the spatial position of the stimuli was varied according to the GellermanN series [12]. Thirty trials were administered daily until subjects responded correctly on 90 out of 100 consecutive trials.

Following the completion of training, the animals received daily equivalence tests, modelled after KLUEvER's procedure [13]. Each equivalence test consisted of 48 trials, on 36 of which the training stimuli were presented in the same manner as they were in prior training. On the remaining 12 trials, the 12 pairs of test stimuli shown in Fig. 1 were presented, and the subject was rewarded for choosing either test stimulus in each pair. One trial in cach of the 12 blocks of 4 trials constituting a test session was randomly selected as a test trial, and the order of presentation of test pairs was randomized. The left-hand members of half the test pairs shown in Fig. 1 were presented on the left on even-numbered test sessions and on the right on odd-numbered test sessions. This rule was reversed for stimuli in the remaining test pairs. If a subject failed to respond correctly on at least $\mathbf{3 2}$ of the 36 training triais in any test session, its performance on test trials in that session was not counted, and it received daily discrimination training, administered as in prior training, until it attained 90 per cent correct responses in one session. On the following day, the subject returned to equivalence testing. All subjects were tested in 20 equivalence sessions in which discrimination performance was 90 per cent correct or better.

\section{RESULTS AND DISCUSSION}

As seen in Fig. 3, both the LS and IT groups required more trials to learn the trianglecircle discrimination than the unoperated controls did. A multiple-comparison t-test (DUNNETT [14]) reveals that the IT monkeys were significantly retarded in discrimination learning compared to the unoperated controls $(t=4.25 ; d f=9 ; p<0.05)$, as were the LS monkeys $(t=2.41 ; d f=9 ; p<0.05)$. On the other hand, the scores of the two operated groups were not reliably different from each other.

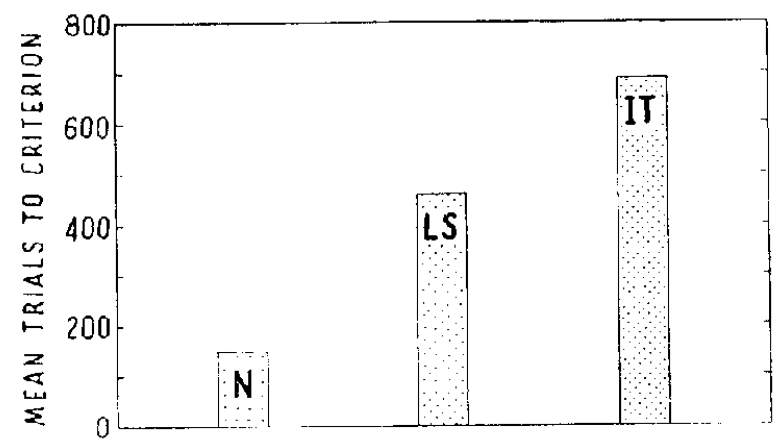

FIG. 3. Mean trials to discrimination learning criterion (not including the last 100 trials) for the normal (N), latcral striate (LS) and inferotemporal (IT) groups (cxp. 1).

The mean preferences of the three groups for the different sets of equivalence stimuli are shown in Fig. 4. With regard to pairs 1-3 (Fig. 4A), the control animals, as well as the IT animals, all chose the forms with the straight line at the bottom (i.e. those with the correct base cue) on virtually all test trials. When presented with pairs $4-6$, in which the base cue was not available, the control animals, like the IT animals, failed to show any consistent preferences for the triangles (see Fig. 4B). When various parts of the training stimuli were removed (pairs 10-12), all animals tended to show weak preferences or no preferences for the triangle in these pairs. Therefore, group preferences were averaged 
over all three pairs in this set (see Fig. 4D). With regard to performance with pairs 7-9 (Fig. 4C), however, reliable group differences were found. While the LS and unoperated animals performed near chance with these stimuli, the IT animals consistently performed

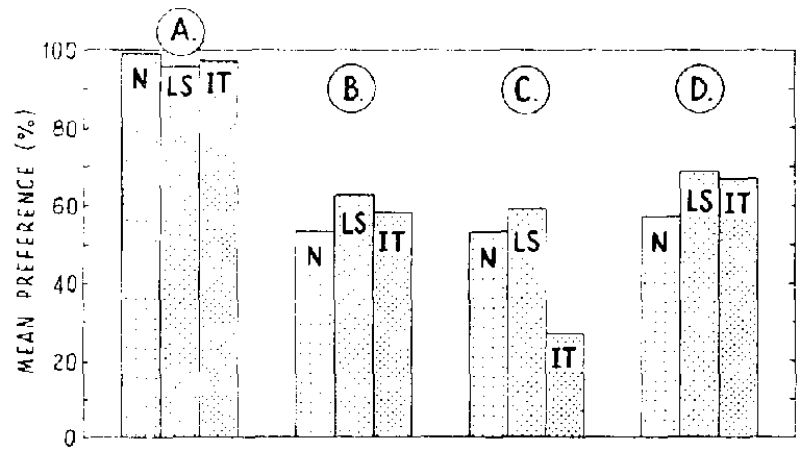

FIG. 4. Mean per cent preferences of the normal (N), lateral striate (LS) and inferotemporal (IT) groups for: A. test pairs I-3; B. test pairs 4-6; C. test pairs 7-9, and D. test pairs 10-12 (exp. 1). In all cases preferences are for left-hand members of test pairs in Fig. 1.

below chance. That is, they preferred the non-triangular forms in these pairs (the forms with the positive base cue) to the triangles. These preferences of the IT monkeys deviated reliably from chance, as indicated by the highly significant $z$-scores $(p<0.01)$ derived from the preferences of all four IT subjects. Furthermore, these preferences of the IT animals were consistent over the 20 test sessions, as were the control animals' lack of preferences for these stimuli. Session by session analyses of preferences for other test stimuli as well, failed to disclose any consistent changes in performance for any of the groups.

Many of the alterations in pattern equivalence that were anticipated were not found in the IT animals, in part, because the control animals' equivalence behavior was more extreme than cxpected. Thus, the control animals' strong preferences for the stimuli with the base cue in pairs 1-3 and their lack of preferences for the triangles in pairs 4-6, precluded the possibility of the IT monkeys showing the more extreme preferences that were expected. On the other hand, the prediction that the IT monkeys would show abnormally strong preferences for the non-triangles in pairs 7-9 was borne out. It is possible that group differences in equivalence were found only when the more and less salient features conflicted with each other, because this conflict situation provides a more sensitive test of the IT animals' alteration than does simply removing cues. In a previous experiment, IT monkeys also showed a similar alteration in equivalence behavior when more and less salient cues conflicted with each other [9]. The equivalence findings, then, in part support the conclusion that the IT monkeys had utilized the base cue to a greater extent than the control animals did in learning the triangle-circle discrimination.

\section{EXPERIMENT 2}

The equivalence behavior which the control animals showed in the first experiment suggests that they had learned little about the specific features of the positive training form in prior discrimination learning. Thus, when the training triangle was paired with other forms in pairs 4-6, the control animals pertormed at chance. The second experiment was undertaken in order to determine whether the control animals would learn to utilize 
the specific features of the training triangle once the differential base cue was no longer available. Furthermore, it was of interest to determine whether the IT monkeys could be trained to use cues other than the base cue in the training triangle; and, if they could learn, whether the effective stimulus would be the same for them as for the control animals.

\section{METHODS}

The animals used in the previous experiment were trained in the WGTA to discriminate between the same triangle used previously (positive) and a straight line which forms the base of that triangle (negative) shown at the top of Fig. 5. The discrimination training procedure was the same as that used in experiment 1 . Following attainment of the discrimination training criterion, the animals were tested for equivalence with the pairs of test stimuli shown in Fig. 5. These test pairs included stimuli presented in the previous test (pairs $1-3$ ), which were included in order to determine how much the animals had learned about the specific features of the triangle in training. The remaining pairs of test stimuli were included in order to determine whether the animals had learned to abstract triangularity independently of the specific features of the training triangle. The equivalence testing procedures were the same as those employed in experiment 1.

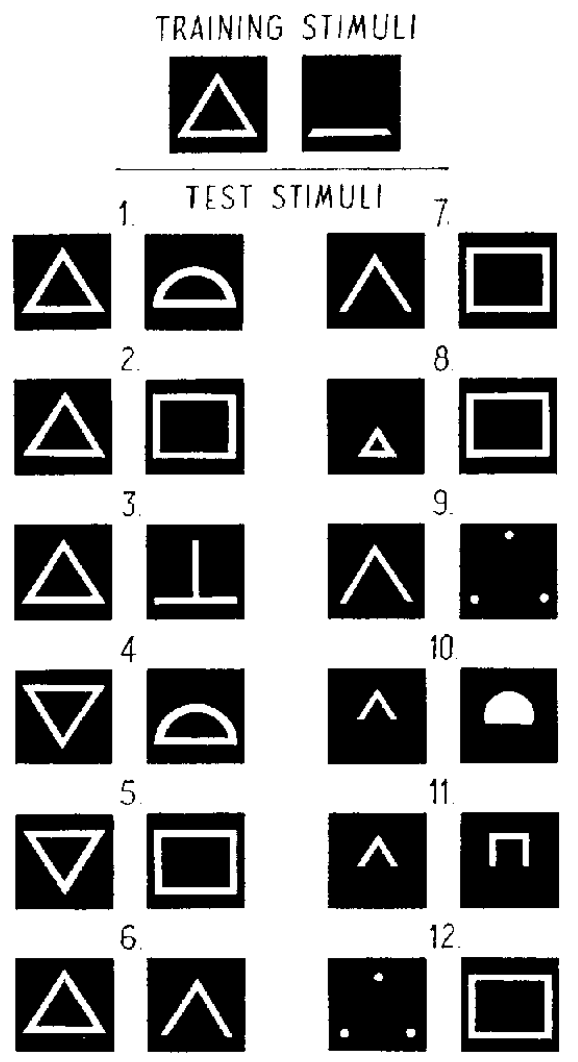

Fig. 5. Stimulus pairs used in discrimination training and in equivalence testing (exp. 2).

\section{RESULTS}

As seen in Fig. 6, the unoperated monkeys learned the discrimination rapidly, and while the LS monkeys as a group required more trials to meet the criterion than did the unoperated monkeys, they were not reliably impaired. The IT monkeys, on the other hand, 


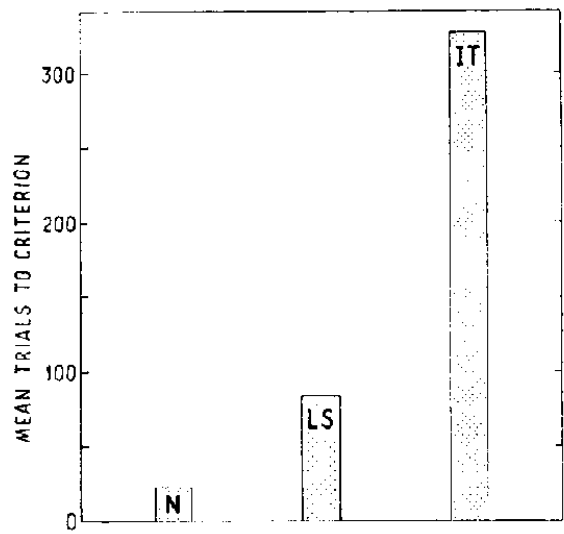

Fig. 6. Mean trials to discrimination learning criterion (not including the last 100 trials) for the normal (N) lateral striate (LS) and inferotemporal (IT) groups (exp. 2).

required significantly more trials to attain the discrimination criterion than did the unoperated monkeys $(t=3 \cdot 14 ; d f=9 ; p<0.05)$. The difference between the learning scores of the IT and LS monkeys approached significance $(t=2.48 ; d f=9 ; p<0.10)$, according to Dunnett multiple-comparison $t$-tests [14].

Turning to the results of equivalence testing, average preferences of the three groups for the triangles in pairs 1-3 are shown in Fig. 7A. While the mean preferences of the control animals for these stimuli were higher than the mean preference of the IT animals, no group differences are significant. Nevertheless, analyses of individual subjects' preferences reveal important group trends. The average preferences of three of the four normal subjects for the triangles in these pairs departed significantly from chance $(p<0.025$ in all cases), while none of the IT subjects showed significant preferences for these triangles. Two of the four LS subjects showed significant preferences for these triangles $(p=0.05$ and $p=0.01$ ). Moreover, the groups differed in the extent to which preferences for these triangles changed from the prior experiment to the present one (see Fig. 7B). All the unoperated animals showed increases in their preferences for triangles. On the other hand,

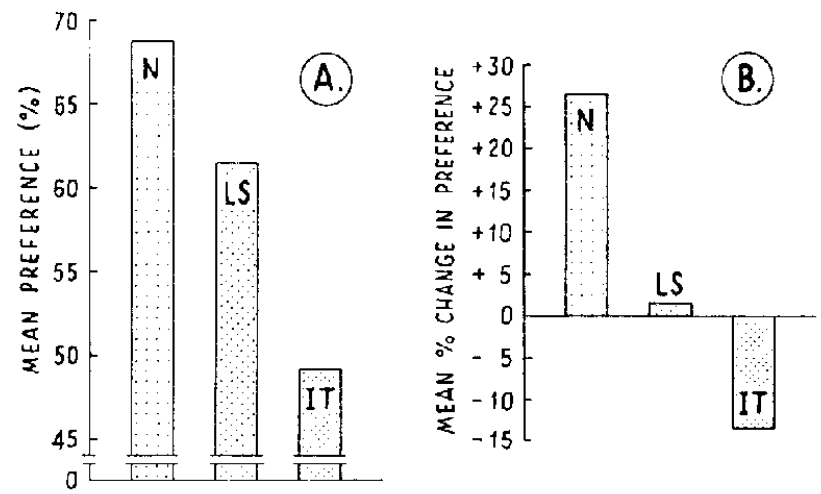

FIG. 7. A. Mean per cent preferences of the normal (N), lateral striate (LS) and inferotemporal (IT) groups for triangles in test pairs 1-3 (exp. 2). B. Mean change from exp. 1 to exp. 2 in per cent preference for triangles in test pairs $1-3$ for the normal (N), lateral striate (LS) and nferotemporal (IT) groups. 
only one IT animal increased its preference, but less than any of the unoperated animals did; one IT animal showed no change, and the two other IT animals showed decreascd preferences. Three of the LS animals showed slight increases in preferences, while one showed a decrease. Dunnett $t$-tests reveal that the normal group's preference increase was significantly greater than that of the IT group $(t=3.94 ; d f=9 ; p<0.01)$. The difference between the preference increase of the normal and LS group approached significance $(t=2.48 ; d f=9$; $p<0 \cdot 10)$.

With regard to the remaining equivalence stimuli, the unoperated monkeys showed no preferences for the triangles in these pairs $(\bar{x}=49.7$ per cent), and the preferences of the LS and IT monkeys for these triangles were slightly below chance $(\bar{x}=42 \cdot 1$ per cent and $\bar{x}=43.9$ per cent respectively). Since the non-triangles in six of these nine pairs were greater in area than the triangles with which they were paired, it is possible that the operated animals were preferring stimuli of larger area. In order to test this hypothesis, average preferences for the larger stimulus in each of the pairs 4-12 were computed for each subject (ignoring differences in configuration). Compared to the mean preference of the normal group ( $\bar{x}=57.0$ per cent), the mean preference of the IT group for the larger stimuli in these pairs $(\bar{x}=74.8$ per cent $)$ was significantly greater $(t=3.56 ; d f=9 ; p<0.05)$, as was the mean preference of the LS group $(\bar{x}=70.4$ per cent) $(t=2.68 ; d f=9 ; p<0.05)$. Finally, analyses of performance over successive test sessions failed to reveal any consistent trends for any of the three groups.

In this experiment, the IT animals were retarded in learning the discrimination, in which the base of the positive triangle was substituted for the negative stimulus (circle) used in experiment 1. This finding provides further evidence that compared to their controls, the IT animals had utilized the base cue to a greater extent in learning the trianglecircle discrimination. Furthermore, once the IT monkeys had learned to choose the triangle without the benefit of the base cue, they behaved in equivalence testing as though they had not identified its specific features, while the unoperated animals apparently had identified these features to some extent. Since the IT subjects had abnormally strong preferences for the larger stimuli in equivalence pairs, it appears that they had learned in prior discrimination training to go to "the larger of two stimuli" and not to "the triangle". Moreover, with regard to the LS animals, the results of equivalence testing suggest that compared to the normal animals, they too, had learned less about the features of the training triangle and had depended on the size cue in discrimination learning.

\section{EXPERIMENT 3}

The final experiment was undertaken in order to determine whether the operated animals could be trained to choose the triangle used in prior discrimination learning when neither the base cue available in experiment 1 nor the size cue in experiment 2 was present. In addition, equivalence tests were administered following discrimination training in order to determine whether and to what extent the operated animals would learn to utilize those stimulus features that the unoperated animals learned to utilize.

\section{ME'THODS}

The same animals used in the previous study were trained in the WGTA to discriminate between the triangle used previously as the positive stimulus, and a U-sliaped figure which was ncgative (see top of Fig. 8). Discrimination training was administered according to the same procedures used in the previous experiments. Following attainment of discrimination criterion, the animals were tested for equivalence, as 
they were previously, with the test pairs shown in Fig. 8. Among these test pairs are four which include the triangle in the same orientation as it was presented in training (pairs 1-4) three of which (pairs 1-3) had been presented in previous tests. The remaining pairs include triangles which differ from the training trianglc. Three of these pairs $(5,6$ and 12$)$ had been presented in previous tests.

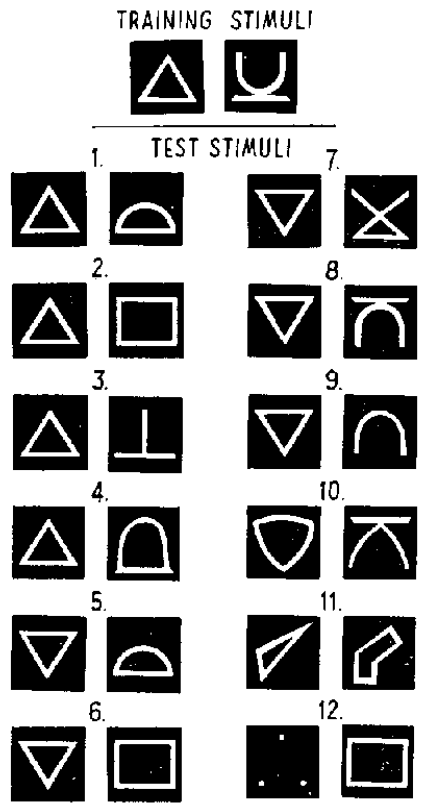

FIG. 8. Stimulus pairs used in discrimination training and in equivalence testing (exp. 3).

\section{RESULTS AND DISCUSSION}

As seen in Fig. 9, the normal group showed very rapid learning of the discrimination problem. On the other hand, all the IT animals required more trials than did the unoperated controls to learn the discrimination. While the learning scores of the normal and IT monkeys do not overlap, the difference between these two groups failed to attain significance when analyzed by a t-test, because of the very large variance of the IT monkeys' scores.

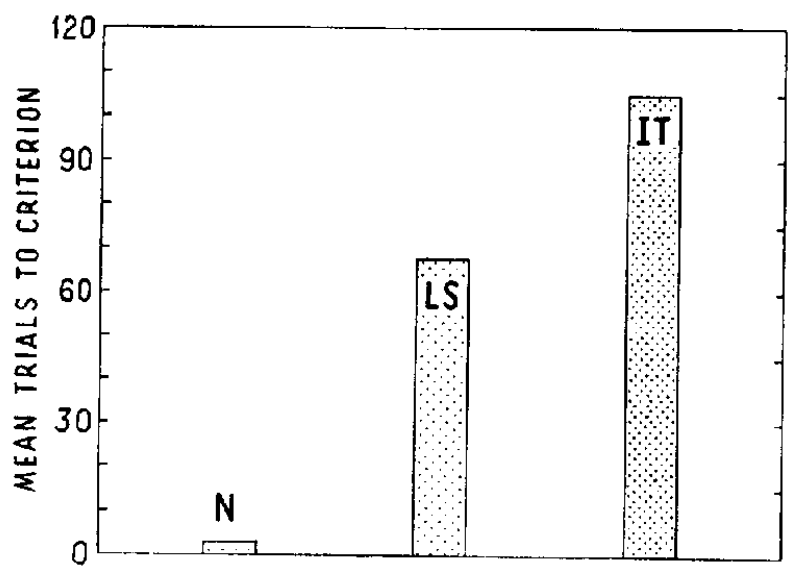

FIG. 9. Mean trials to discrimination learning criterion (not including last 100 trials) for the the normal (N), lateral striate (LS) and inferotemporal (IT) groups (exp. 3). 
With regard to the LS group, their mean learning score fell between those of the other two groups (see Fig. 9). Two LS subjects attained criterion in O trials, while the scores of the other two (57 and 145) fell outside of the normal range.

The results of equivalence testing, shown in Fig. 10, indicate that for the first time, the IT animals were showing normal perceptual equivalence. Considering first preferences for the triangles in pairs 1-3 (Fig. 10A), all animals showed scores which were above chance and larger than the scores they attained in experiment 2. The IT monkeys showed the largest increases in preferences, and, as seen in Fig. 10A, their preference scores were as high as those of the unoperated monkeys. The LS monkeys' preferences for triangles in pairs 1-3 were not reliably lower than those of the other two groups. Sccondly, an analysis of preferences for triangles different from and the same as the training triangle is presented in Fig. 10B. All animals showed stronger preferences for "same" as opposed to

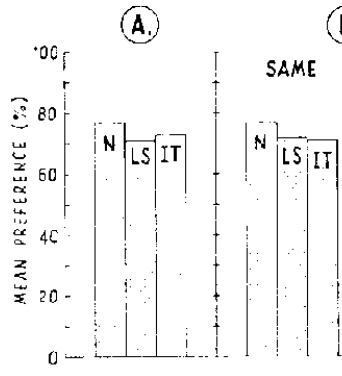

(B.)

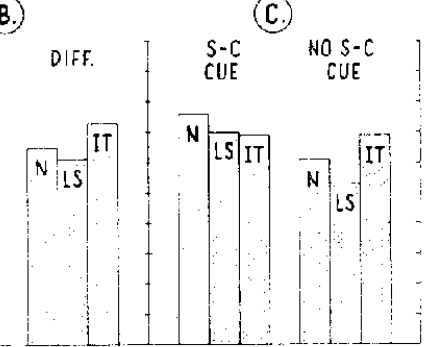

FIG. 10. Mean per cent preferences of the normal (N), lateral striatc (LS) and inferotemporal (IT) group for: A. triangles in test pairs 1-3; B. triangles identical to the training triangle (in pairs 1-3,6), and triangles different from the training triangle (in pairs 4, 5, 7-12); $\mathrm{C}$. triangles in test pairs containing straight vs. curved border cues (in pairs $1,4,6,8-10$ ), and triangles in test pairs not containing straight vs. curved border cues (in pairs $2,3,5,7,11,12$ )

(exp. 3).

"different" triangles. However, all the IT animals, as well as the unoperated animals, did show significant preferences for different triangles, and, as seen in Fig. 10, the IT animals on the average showed somewhat stronger preferences for these triangles than did the unoperated animals. Only two of the four LS animals showed significant preferences for different triangles; however, as a group the LS animals did not differ from the others in their preferences for these triangles. Finally, an analysis of preferences for straight vs. curved border cues is presented in the right panel of Fig. 10. The discrimination task in this experiment could have been learned on the basis of straight borders (in the positive triangle) vs. curved borders (in the negative U-shaped form), rather than on the basis of configurational differences. Thus, preferences for triangles in test pairs which do not contain this cue might also provide a measure of the animals' ability to utilize configuration cues. As seen in the right panel of Fig. 10, the normal and LS groups showed stronger preferences for triangles when this cue was available than when it was not, a difference which the IT group did not show. Furthermore, as seen in Fig. 10, the IT group showed the highest mean preference scores for triangles without the straight-curved border cue. All four IT monkeys showed significant preferences for the triangles in these pairs $(p=0.03$ and less), while only two of the normal monkeys ( $p=0.05$ and 0.005$)$ and one LS monkey $(p=0.005)$ showed significant preferences for these triangles. As in the prior experiments, there were no consistent changes in preferences for test stimuli over the 20 equivalence sessions for any of the groups. 
The IT monkeys' discrimination learning deficit might be attributed to their having learned in experiment 2 to avoid the smaller, negative stimulus, for which the U-shaped form was substituted in the present experiment. A similar argument could be advanced with respect to the IT animals' discrimination learning deficit in experiment 2 . However, it does not appear that the IT monkeys had a generalized deficit in learning to approach positive stimuli in discrimination learning. In experiment 1 the IT animals all showed strong tendencies to choose the positive base cue even when the negative base cue was not present (pairs 8 and 9). Thus, their subsequent impairment in choosing the triangle paired with the triangle base (exp. 2) cannot be readily attributed to a failure to learn to approach the triangle. Furthermore, in the present experiment, the IT monkeys were not impaired in choosing triangles paired with novel forms which differed from the negative, U-shaped stimulus used in training. It is more likely, then, that the IT animals' discrimination learning deficit in the present experiment was due to a deficiency in identifying the specific features of the training triangle in experiment 2 , an interpretation which is consistent with their altered equivalence behavior in that experiment.

However, once the IT monkeys succeeded in discriminating the triangle from the Ushaped figure, their performance in equivalence testing suggests that they had learned as much about the features of the training triangle as the normal animals did. Thus, the IT animals apparently identified the specific aspects of the training triangle as well as the unoperated animals did, as their performance with pairs 1-3 would seem to indicate. Furthermore, it appears that the IT animals also abstracted triangularity at least as well as the normal animals did, for they showed better than normal preference for triangles different from the training triangle and triangles that could not be identified on the basis of straight borders. Alternatively, it is possible that the IT monkeys' improvement in equivalence was due simply to the extensive discrimination training that they received over the three experiments, including test sessions. However, this interpretation is inconsistent with the finding that these animals, like their controls, showed no improvement in equivalence within test sessions in any of the experiments.

The normal equivalence behavior of the IT animals in the present experiment contrasts with their performance in prior equivalence tests in this study. Thus, after discriminating the triangle from the circle (exp. 1), the IT animals showed an abnormally strong tendency to choose forms containing the base cue rather than those with the configurational cue. This finding, together with their subsequent deficit in discrimination learning when the base cue was removed (exp. 2) supports the view that the IT animals had utilized the base cue to a greater extent than had the control animals. Moreover, after discriminating the triangle from the triangle base, the IT monkeys again showed altered equivalence in experiment 2. These alterations in equivalence suggested that compared to the unoperated controls, they had failed to identify the particular features of the training triangle and utilized the height cue to a greater extent. The finding that the IT monkeys were impaired in the subsequent discrimination, in which the height cue was removed (exp. 3), is consistent with this interpretation. These findings strengthen the hypothesis based upon previous results $[4,5]$ that IT monkeys are deficient in detecting the less salient aspects of visual stimuli.

According to the findings obtained in the last experiment, IT animals can apparently overcome this deficit and identify the less salient features of patterns once they are required to discriminate patterns without the benefit of the more distinctive base or height cues. The IT monkeys' eventual success in abstracting triangularity suggests that these animals 
are capable of normal perceptual identification once they attend to the appropriate stimulus features. In other words, it appears that the IT monkeys' impairment in equivalence, like their impairment in generalization [8], is not due to a defect in coding perceptual features. Rather, the present findings suggest that their impairment may be due to a defect in sampling stimuli or attending to several aspects of stimuli. For, identification of the less salient configurational cue requires attending to several parts of the form, whereas identification of the base or height cue requires attending only to a single feature.

This interpretation of the present findings implies that the IT monkey's visual learning deficit, like their equivalence impairment, is not due to a loss in the capacity to identify the features of visual stimuli, even complex features (i.e. triangularity). Rather, the analysis presented here implies that the visual learning deficits might be due to a deficiency in sampling or selectively attending to various aspects of visual stimuli. PRIBRAM [15] has offered a similar interpretation of deficits in multiplc-object discrimination learning. In that experiment, the IT monkeys seemed to be impaired in sampling among several objects. Moreover, recent electrophysiological data suggest that IT cortex is selectively involved in the regulation of visual input [16], a role which is consistent with the present interpretation of deficits consequent to IT removal. However, there are behavioral findings $[17,18]$ which appear to be at variance with the interpretation of the underlying disturbance offered here. In both of these experiments, IT monkeys were first trained to discriminate between two stimuli (in one case, stimuli differing in size; in the other, flickering vs. steady light). The IT animals subsequently showed impaired performance when stimulus differences were gradually reduced, even though they had clearly attended to the relevant stimulus dimensions in initial training. It is not unlikely, however, that increasing the difficulty of sensory discriminations in this manner places increasing demands on stimulus sampling and attention, which cannot be met by IT monkeys. With respect to this suggestion, it should be noted that in the study employing flickering vs. steady light discrimination [18], the stimulus was spatially separated from the site of the response, a condition which is likely to lead to decreased "stimulus orienting" [19]. In a more recent experiment [20], in which there was no stimulus-response discontiguity, IT monkeys did not show deficits in flicker vs. steady light discrimination.

With regard to the LS monkeys, these animals showed some partial deficits similar to those of the IT monkeys in the first two experiments. Thus, the LS group was impaired in learning the initial triangle-circle discrimination, but, unlike the IT group, showed no alterations in subsequent equivalence tests in that experiment. Furthermore, two LS subjects-those that were more severely impaired in the initial discrimination task-were the only animals in that group impaired in the discrimination task in experiment 2 . The same LS animals also showed alterations in the subsequent equivalence tests in experiment 2; like the IT animals, they failed to show preferences for the training triangles in pairs 1-3, and they showed abnormally strong preferences for the larger stimuli in test pairs. Furthermore, these two LS monkeys were also impaired in learning the final discrimination problem. Thus, with regard to these findings, the LS monkeys' impairment appears similar to that of the IT monkeys; those LS animals that were retarded in learning also showed equivalence alterations like those of the IT subjects. However, unlike the IT animals, the two LS animals impaired in the first two experiments showed abnormally low preferences for triangles in the final series of equivalence tests. Apparently, they had not learned to utilize the specific features of the training triangle or to abstract triangularity, as the IT monkeys did. This finding suggests that the LS animals' equivalence impairment is not similar 
to that of the IT animals, but is qualitatively different. This equivalence impairment might possibly be attributed to visual field defects, which may have interfered with the LS monkeys' detection of patterns used in these experiments. Alternatively, it is conceivable that the pattern equivalence impairment of the LS animals may be due to a loss in perceptual integration, perhaps similar to those described in humans with lesions of the geniculostriate system [21, 22].

\section{REFERENCES}

1. BRown, T. S. Olfactory and visual discrimination in the monkcy after selective lesions of the temporal lobe. J. comp. physiol. Psychol. 56, 764-768, 1963.

2. Weiskrantz, L. and Mishrin, M. Effects of the temporal and frontal cortical lesions on auditory discrimination in monkeys. Brain 81, 406 414, 1958.

3. WILsON, M. Effects of circumscribed cortical lesions upon somesthetic and visual discrimination in the monkey. J. comp. physiol. Psychol. 50, 630-635, 1957.

4. Cowey, A. and Weiskrantz, L. A perimetric study of visual field defects in monkeys. Quart.J. exp. Psychol. 15, 91-115, 1963.

5. Wfiskrantz, L. and Cowey, $\Lambda$. Striate cortex lesions and visual acuity of the rhesus monkey. J. comp. physiol. Psychol. 56, 225-231, 1963.

6. WILSON, W. A. JR. and MishKIN, M. Comparison of the effects of inferotemporal and lateral occipital lesions on visually guided behavior in monkeys. J. comp. physiol. Psychol. 52, 10-17, 1959.

7. Mishкin, M. Visual functions beyond striate cortex. In Frontiers of Physiological Psychology, R. W. Russell (Editor), pp. 93-116, Academic Press, New York, 1966.

8. Butter, C. M., Mishkin, M. and Rosvold, H. E. Stimulus generalization in monkeys with inferotemporal and lateral occipital lcsions. In Stimulus Generalization, D. J. MostofsKy (Editor), pp. 119-133, Stanford University Press, Stanford, 1965.

9. Butter, C. M. and Gekoski, W. L. Alterations in pattern equivalence following inferotemporal and lateral striate lesions in rhesus monkeys. J. comp. physiol. Psychol. 61, 309-312, 1966.

10. Butter, C. M. Effective stimuli for pattern discrimination in monkeys. Psychon. Sci. 2, 325-326, 1965.

11. Von Bonin, G. and Bailey, P. The Neocortex of Macaca Mulatta, R. A. Allen, O. F. Dampmeier, I. Schour, E. R. Skrles (Editors). The University of lllinois Press, Urbana, 1947.

12. Gellerman, L. W. Chance orders of alternating stimuli in visual discrimination experiments. J. genet. Psychol. 42, 207-208, 1933.

13. Kluever, H. Behavior Mechanisms in Monkeys, University of Chicago Press, Chicago, 1933.

14. Dunnett, C. W. A multiple comparison procedure for comparing several treatments with a control. J. Am. statist. Ass. 50, 1096-1121, 1955.

15. Pribram, K. H. The intrinsic systems of the forebrain. In Handbook of Physiology, Vol. 2, J. Field H. W. Magoun and V. E. Hall (Editors), pp. 1595-1668. American Physiological Society, Washington, D.C., 1960.

16. Spinelli, D. N. and Pribram, K. H. Changes in visual recovery functions produced by temporal lobe stimulation in monkeys. EEG Clin. Neurophysiol. 20, 44-49, 1966.

17. MishKIN, M. and Har.L, M. Discrimination along a size continuum following ablation of the inferior temporal convexity in monkeys. J. comp. physiol. Psychol. 48, 97-101, 1955.

18. Mishkin, M. and Weiskrantz, L. Effects of cortical lcsions in monkeys on critical flicker fusion. J. comp. physiol. Psychol. 52, 660-666, 1959.

19. Polidora, V. J. and Fletcher, H. F. An analysis of the importance of S-R spatial contiguity for proficient primate discrimination performance. J. comp. physiol. Psychot. 57, 224-230, 1964.

20. Symmes, D. Flicker discrimination by brain-damaged monkeys. J. comp. physiol. Psychol. 60, 470-473, 1965.

21. Teuber, H.-L., Battersby, W. S. and Bender, M. B. Performance of complex visual tasks after cerebral lesions. J. nerv. ment. Dis. 114, 413-429, 1951.

22. Teurfe, H.-L. and WeINSTEIN, S. Performance on a formboard-task after penetrating brain injury. J. Psychol. 38, 177-190, 1954. 
Résumé--Des singes avec lésions inférotemporales (IT), des singes avec lésions du cortex occipital strié latéral (LS) et des contrôles non opérés avaient á discriminer entre 2 modèles, et l'équivalence perceptive était également testée dans 3 séries d'expériences. Les singes IT semblaient utiliser plus de repères marquants que les animaux de contrôle et échouaicnt à identifier les traits spécifiques des modèles (exp. 1 et 2). Quand on supprimait les repères marquants (exp. 3), les animaux IT se révèlaient capables d'identifier normalement les repères configurationnels. Tandis que 2 singes LS montraient des déficits analogues á ceux des singes IT (Exp. 1 et 2), ils n'identifiaient pas les traits configurationnels (exp. 3). Ces constatations suggèrent que les lésions IT ne perturbent pas la capacité d'identification des caractéistiques visuelles, mais perturbent la sélection des stimulus ou les processus attentionnels.

Zusammenfassung -...Affen mit Läsionen im unteren Temporallappen (IT), Affen mit Läsionen in der lateralen Sehrinde (LS) und nicht operierte Kontrolltiere unterschieden 2 Muster und wurden auf die Gleichwertigkeit von Mustern in 3 Versuchen getestet. Die IT-Affen schienen eher als die Kontrolltiere wesentliche Hinweise zu verwenden. Sie versagten bcim Identifizieren von spezifischen Zügen der Muster (Versuche 1 und 2). Wenn wesentliche Anhaltspunkte entfernt wurden (Versuch 3), zeigten sich bei den IT-Ticren zeichen für eine normale Identifikation von Hinweisen in den Figuren. Während 2 LS-Affen ähnliche Ausfälle wie die der IT-Affen zeigten (Versuche 1 und 2), identifizierten sie Züge der Figuren anscheinend nicht (Versuch 3). Die Befunde legen nahe, daß IT-Läsionen nicht die Fähigkeit der visuellen Identifikation beeinträchtigen, sonđern die Reizaufnahme stören oder die Aufmerksamkeit unterbrechen. 